\title{
Chancen und Möglichkeiten der minimal invasiven Chirurgie in der Onkologie
}

\author{
Reinhard Bittner $^{\mathrm{a}} \quad$ Eckhard Bärlehner $^{\mathrm{b}} \quad$ Jürgen F. Riemann ${ }^{\mathrm{c}}$ \\ ${ }^{a}$ Klinik für Allgemein- und Visceralchirurgie, Marienhospital Stuttgart \\ ${ }^{b}$ Chirurgische Klinik, Zentrum für Minimal Invasive Chirurgie, HELIOS Klinikum Berlin-Buch \\ ${ }^{\mathrm{c}}$ Medizinische Klinik C, Klinikum der Stadt Ludwigshafen, Deutschland
}

Minimal invasive Therapie in der Onkologie bedeutet zum einen Minimierung des Zugangstraumas bei ansonsten identischer onkologischer Radikalität wie in der herkömmlichen Chirurgie, zum anderen aber auch die lokale Exzision einer prämalignen oder malignen Läsion unter Verzicht auf ein größeres Organopfer. Die Minimierung des Zugangstraumas wurde möglich durch die Einführung der laparoskopischen Operationstechnik. Deren Grundprinzip ist die Schaffung eines freien Raums in der Bauchhöhle durch Anlage eines Pneumoperitoneums und die Durchführung der einzelnen Operationsschritte unter videoendoskopischer Kontrolle. Während diese Technik bei der Entfernung der Steingallenblase aufgrund der hohen Akzeptanz durch die Patienten weltweit rasch Eingang in die Klinik fand, waren in der onkologischen Chirurgie zunächst zwei Fragen zu klären:

- Welchen Einfluss hat das Pneumoperitoneum auf die Verbreitung von Tumorzellen, und besonders auf die Entstehung von so genannten Trokarkanalmetastasen?

- Lassen sich die standardisierten und in der offenen Chirurgie bewährten Operationsschritte in gleicher Weise auch laparoskopisch durchführen?

Mit dem vorliegenden Heft der Chirurgische GastroENTEROLOGIE INTERDISZIPLINÄR soll die Diskussion dieser wichtigen Fragen weiter vorangetrieben und dem praktisch tätigen Chirurgen aktuelle Informationen für die tägliche Entscheidungsfindung zur Hand gegeben werden.

Wie sich mittlerweile gezeigt hat, führt weder das Pneumoperitoneum zu einer verstärkten Tumorzellausbreitung - im Besonderen in der Bauchhöhle -, noch wird die onkologische Radikalität durch die minimal invasive Therapie kompromittiert. Nach den vor kurzem publizierten Langzeitergebnissen der ersten großen randomisierten Studie (COST-Studie) kann mit der laparoskopischen Technik bei der Resektion eines Kolonkarzinoms das gleiche onkologische Spätergebnis erzielt werden wie mit der konventionellen chirurgischen Technik, vorausgesetzt der Chirurg verfügt über die Erfahrung von mindestens 20 laparoskopischen Kolonresektionen wegen einer benignen Erkrankung. Trotzdem sind nach wie vor viele Fragen offen, im Besonderen können durch das reduzierte Zugangstrauma sowie einer weniger traumatisierenden Operationstechnik die Immunreaktion des Körpers verbessert und somit letztlich auch gewisse Vorteile für das Langzeitergebnis erzielt werden.

Der zweite Problemkomplex, der in diesem Heft eingehend beleuchtet werden soll, betrifft die minimal invasive Therapie von prämalignen und malignen Läsionen per se. Das heißt, es wird der Frage nachgegangen ob eine lokale Exzision zum gleichen onkologischen Ergebnis führt wie eine radikale Operation mit einem mehr oder weniger großem Organopfer. Es besteht kein Zweifel, dass eine maligne Läsion durch eine lokale Exzision - sei sie endoskopisch oder laparoskopisch durchgeführt - radikal beseitigt werden kann, vorausgesetzt dass ein bestimmtes Stadium der Tumorerkrankung nicht überschritten ist. Welche Faktoren hierfür entscheidend sind Umfang der Läsion, Eindringtiefe in die Wand des Gastrointestinaltrakts, Grading, die Mitbeteiligung von Lymph- und Blutgefäßen - wird in den Beiträgen des vorliegenden Hefts dargestellt.

Die Verbesserung des Stellenwerts der minimal invasiven Chirurgie in der Onkologie wurde auch durch die Weiterentwicklung der diagnostischen Verfahren und endoskopischen Therapiemaßnahmen ermöglicht. Nicht zuletzt aufgrund dieser neuen Therapieoptionen ist eine enge $\mathrm{Zu}$ sammenarbeit zwischen Gastroenterologen und Viszeralchirurgen mehr denn je erforderlich. Nur in der kontinuierlichen interdisziplinären Diskussion einschließlich gemeinsamer klinischer Studien können zum Wohle des Patienten die Vor- und Nachteile der minimal invasiven Therapie bestimmt werden.

\begin{tabular}{ll}
\hline KARGER & @ 2005 S. Karger GmbH, Freiburg \\
Fax +49 7614520714 & Accessible online at: \\
$\begin{array}{l}\text { E-mail Information@Karger.de } \\
\text { www.karger.com }\end{array}$ & www.karger.com/cga
\end{tabular}

